\title{
Cannibalism facilitates the use of a novel environment in the flour beetle, Tribolium castaneum
}

\author{
SARA VIA* \\ Department of Biology and Department of Entomology, University of Maryland, College Park, MD 20742, U.S.A.
}

\begin{abstract}
Cannibalism is well known to affect both the population dynamics and the competitive relationships of organisms. Cannibalistic behaviour commonly increases in stressful conditions, such as when density is high or food is scarce, and cannibals often obtain a nutritional benefit. Might cannibalism also increase in a novel environment to which a population is poorly adapted physiologically? Moreover, might cannibalistic behaviour provide enough of a nutritional advantage in a nutritionally stressful environment to rescue individuals from its adverse effects and thus permit colonization and range expansion? Previous work has shown that oat flour is a particularly stressful environment for Tribolium castaneum. In the study reported here, egg cannibalism by two strains of T. castaneum was significantly enhanced in oat flour, and egg eating rescued larvae from the adverse demographic effects of this poor environment. Development time of the cannibals was accelerated almost to the level seen for individuals reared in the nutritionally superior environment (wheat plus brewer's yeast). Their survival and fecundity also increased relative to individuals reared in oat flour without the opportunity to cannibalize. A sib analysis revealed that for larvae reared in the presence of victim eggs, the extent of cannibalism was genetically variable, so that this trait could evolve, given a selective benefit exceeding its cost. These results suggest that colonization of a marginal new environment could be facilitated by enhanced rates of cannibalism. The possible interplay between cannibalism and physiological adaptation to a new environment is discussed.
\end{abstract}

Keywords: colonization, ecological genetics, evolution, insects, quantitative genetics, resource use.

\section{Introduction}

Cannibalism occurs in a wide range of organisms and is thought to be a common feature of many life cycles (Fox, 1975; Polis, 1981). The ecological effects of cannibalism are clear. Predation on conspecifics can significantly affect population size and age structure, and it is often implicated as a mechanism of population regulation (Park et al., 1965; Lloyd, 1968; Van Buskirk, 1989; Fincke, 1994; Wagner \& Wise, 1996). Cannibalism can alter competitive relationships both by eliminating competitors and by providing the cannibal with nutritional benefits (Fox, 1975; Johansson, 1992; Fincke, 1994). The nutritional benefit of cannibalism need not be large for the behaviour to evolve: even a small decrease in the development time of the cannibal in a species with overlapping generations may provide

*E-mail:sv47@umail.umd.edu enough of a selective advantage to cause the spread of cannibalism, even of full-sibs (Eickwort, 1973).

Evidence that cannibalism is nutritionally beneficial has come both from direct observations of improved survival, development time or fecundity of cannibals (Ho \& Dawson, 1966; Fox, 1975; Sonleitner \& Guthrie, 1991; Spence \& Carcamo, 1991; Agarwala \& Dixon, 1992; Tschinkel, 1993), and from the observation that cannibalism often increases when alternative food is scarce (Fox, 1975; Polis, 1981; Naseer \& Abdurahiman, 1993). This has led some authors to suggest that cannibalism may permit populations to persist through stressful periods (Agarwala \& Dixon, 1992; Naseer \& Abdurahiman, 1993; Watanabe \& Yamaguchi, 1993; Parajulee \& Phillips, 1995).

There is a related possibility, that cannibalistic behaviour might facilitate colonization of new environments by providing a nutritional benefit that compensates for a food supply that is marginal for an unadapted population. This is a largely unexplored ecological and evolutionary issue (but see Johansson, 
1996; Wissinger et al., 1996). Evaluating this possibility was the goal of this study, in which the flour beetle, Tribolium castaneum, was used as a model system.

The study of cannibalism and its effects on population dynamics and competitive interactions in Tribolium has a long history (Sonleitner, 1961; Park, 1948, 1962; Park et al., 1964, 1965; Lloyd, 1968; Stevens, 1989). In Tribolium, both adults and larvae eat eggs (Rich, 1956; Sonleitner, 1961). Pupae are also sometimes consumed (Park et al., 1968). Cannibalism is a potent force in the regulation of Tribolium populations, and it mediates the interspecific competition between $T$. castaneum and T. confusum (Sokoloff et al., 1965). Strains of Tribolium differ genetically in their tendency toward cannibalistic behaviour, and the most cannibalistic strains maintain a much lower population size than do less cannibalistic strains (Park et al., 1965; Stevens, 1989).

It is sometimes stated or assumed (e.g. Stevens, 1989; Hastings \& Costantino, 1991) that cannibalism provides no individual advantage to a Tribolium larva in a high quality environment such as the 'standard medium' (whole wheat flour with 5\% brewer's yeast, henceforth 'wheat5'). However, several studies have reported nutritional benefits from egg eating in wheat5. Ho \& Dawson (1966) reported a significant increase in the fecundity of adults that had been allowed to eat eggs as larvae. Mertz \& Robertson (1970) report that larvae were rescued from the detrimental effects of high density when they were allowed to eat eggs.

Although most previous studies of cannibalism in Tribolium have been restricted to studies of populations in the standard medium (wheat5), Tribolium is a common pest of many types of grains, most of which are lower in nutritional quality than is wheat5 (Sokoloff et al., 1966a,b). These grains constitute stressful environments for nonadapted populations. Via (1991) showed that both development time and populationlevel productivity were lowered in larval groups reared in maize, rice or oat flour, with oat being a particularly poor environment. Dawson (1968) studied T. castaneum in maize flour and suggested that larval survival may be enhanced by the opportunity to consume eggs of the related species $T$. confusum.

The study reported here was undertaken to determine both whether cannibalistic behaviour might be enhanced in oat flour, and whether it might boost individual performance in this poor environment as Mertz \& Robertson (1970) found in the high density situation. Several different experiments were performed in order to analyse the extent and benefits on growth of egg eating in oat flour relative to growth in the standard medium and to estimate genetic variability in cannibalism in different flour types (as suggested by work of Wade (1980) and Stevens (1989) in wheat5). Together, these experiments reveal that cannibalism in oat flour is enhanced, and that it does rescue populations from the adverse physiological effects of this novel environment. Moreover, estimates of genetic variability within both strains in oat flour suggest that altered cannibalism rates could potentially evolve in populations colonizing this nutritionally stressful environment.

\section{Background and methods}

\section{Performance of two strains of Tribolium castaneum in dierent environments}

The flour beetle, Tribolium castaneum, infests many types of stored grains (Sokoloff et al., 1966a). In the experiments reported here, cannibalism rates on different grains were tested for two strains of $T$. castaneum. Most of the experiments were performed on 'ChicagocSM', a strain synthesized in 1978 by Dr Michael J. Wade at the University of Chicago from several of Dr Thomas Park's inbred strains. The second strain is called 'Purdue +2 ', and was synthesized in 1984 by $\mathrm{Dr}$ A. E. Bell by crossing two independent lines of Purdue + , originally cultured in 1954 from field-collected beetles. Because both the strains used here (and their immediate progenitor strains) have been maintained continuously in the laboratory on standard medium (wheat5) for several hundred generations, other flours can reasonably be assumed to be novel environments for these strains.

Via (1991) exposed replicated groups of beetles to five different flours (wheat5, unsupplemented wheat, maize, rice, and oat), and showed that development time for Chicago-cSM on oat flour took an average of 16.5 days $(58 \%)$ longer than on wheat5, and that the mean population size was greatly reduced in oat flour relative to wheat 5 . The reduced population size was shown to be caused by a combination of reduced oviposition, cannibalism, and increased larval mortality. For Purdue +2 , oat flour was not as poor an environment. For that strain, development took only about 12 days longer than on wheat5, with no statistically significant difference in population sizes in the two environments (Via, 1991).

The survivorship of Chicago-cSM larvae raised as individuals in different flours was tested in a sibling analysis (Via \& Conner, 1995). By rearing progeny of 20 half-sib families individually, the effects of poor nutritional quality of a given flour on growth and development were separated from other causes of poor performance in the novel flour environments. A previously unreported result of that experiment is that the mean mortality of individuals raised in oat flour was significantly greater than that of individuals reared in other flours $(42 \%$ mortality on oat, $9 \%$ on wheat5, Fig. 1). 
Thus, oat flour appears to be an environment of marginal suitability for these two strains of $T$. castaneum, and it is a particularly poor resource for Chicago-cSM.

\section{Experiment 1. Relative cannibalism rates of Chicago-cSM on eggs and pupae}

To determine the relative extent of cannibalism on different life stages, groups of 20 eggs from the stock cultures were allowed to grow into intermediate sized larvae in $10 \mathrm{~g}$ of either the standard environment, wheat5 (15 days), or the stress environment, oat (25 days). These larvae (the cannibals) were then provided with either 60 eggs or 25 pupae. After $48 \mathrm{~h}$, vials were sifted and the cannibals and remaining victims were counted. All treatments were replicated five times. To account for the greater mortality in groups reared in oat flour, cannibalism rates were calculated on a per capita basis for the cannibal larvae remaining at the time of the trial.

\section{Experiment 2. Relative egg cannibalism by Chicago-cSM in four flours}

To compare rates of egg cannibalism in different flour types with a known range of suitability for Chicago-

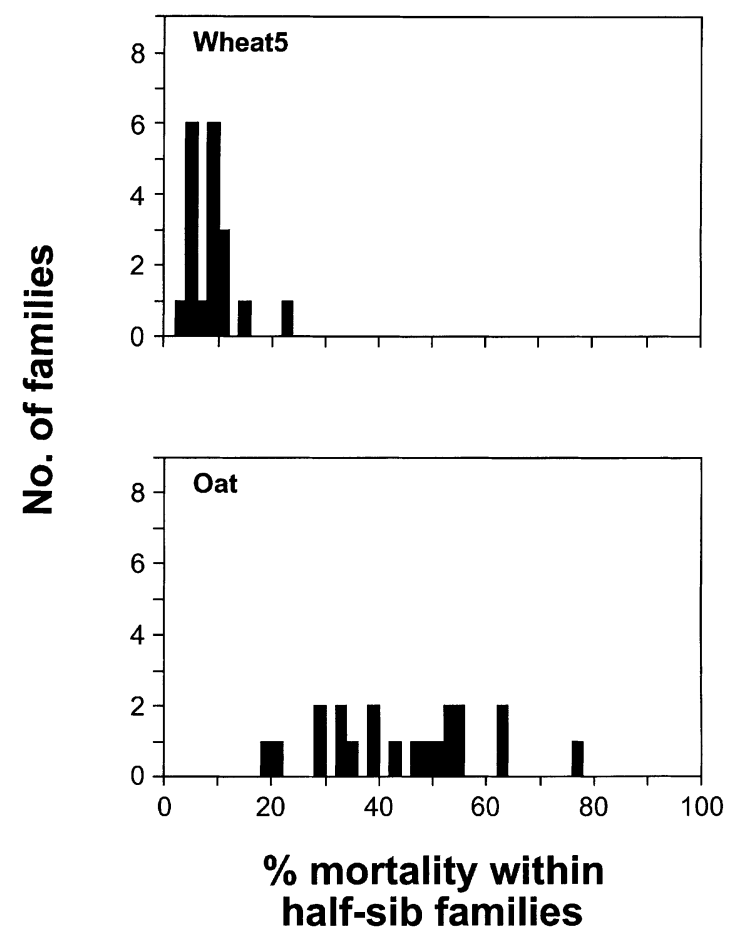

Fig. 1 Distributions of larval mortality for half-sib families of the strain Chicago-cSM Tribolium castaneum reared in either wheat flour with $5 \%$ brewer's yeast (wheat5) or oat flour.
cSM (Via, 1991; Via \& Conner, 1995), groups of 20 randomly chosen eggs from the stock cultures were allocated to vials containing $10 \mathrm{~g}$ of finely sifted wheat5, maize, rice or oat flour (six replicates). After 15 days (wheat5), 20 days (maize and rice) or 25 days (oat), 60 food eggs were added to each vial. Two days later, vials were sifted, and the cannibal larvae and remaining victim eggs were counted.

\section{Experiment 3. Can groups of Chicago-cSM reared in oat flour be rescued by the opportunity to cannibalize?}

To determine whether cannibalism provides a nutritional benefit that might increase performance in oat flour, 20 full-sibling families were created by mating males and females chosen at random as pupae from the stock culture of Chicago-cSM. Six replicate groups of four eggs from a given family were exposed to each of three treatments: wheat5, oat $(\mathrm{O})$, and oat to which 50 eggs were added each week as victims $(\mathrm{O}+\mathrm{E})$. Beginning at day 20, individuals that pupated were removed and checked three times each day for eclosion. For each vial, survivorship, average development time and minimum development time (date on which first individual in a given vial eclosed) were calculated. To evaluate the extent of genetic variability for egg eating, each of the $\mathrm{O}+\mathrm{E}$ vials was sifted two days after addition of the third group of victim eggs on day 21 to evaluate how many eggs were left. Variation in egg eating was partitioned using ANOvA in order to separate variation among full-sib families (total genetic variation) from that attributable to random variation in the environment (e.g. Falconer, 1989). Finally, the first female to eclose from each vial was allowed to mature for 2 days, and was then mated to a male chosen at random from another family for 3 days. Her fecundity was then measured for two successive 3-day periods.

\section{Experiment 4. Cannibalism in four flours by Purdue +2}

To evaluate the relative cannibalism rates of the Purdue strain in different grains, replicate groups of 20 eggs produced by a group of randomly chosen adults from the Purdue +2 culture were allocated to vials of sifted wheat5, wheat, maize, rice and oat flour. Three replicates of each treatment were established. The larvae from these eggs were allowed to grow to approximately the same size in each flour. After 15 days (wheat5), 20 days (maize and rice) or 25 days (oat), 60 food eggs (from Purdue +2 stock cultures) were added to each vial. After 2 days, the remaining cannibal larvae and food eggs were sifted out and 
counted. Cannibalism rate was calculated for each vial on a per-capita basis.

\section{Experiment 5. Genetic variation in egg eating within Purdue +2 for larvae reared without the opportunity to cannibalize}

A sibling analysis was performed in which 20 randomly chosen Purdue +2 males were each mated to four randomly chosen females. Groups of 10 eggs from each mating were allocated to $5 \mathrm{~g}$ of wheat5, maize or oat (two replicates/female/treatment). Larvae were allowed to grow for 14 days (wheat5), 20 days (maize) or 28 days (oat) before 60 victim eggs were added to each vial. After 2 days, the remaining cannibal larvae and victim eggs were sifted out and counted.

A nested analysis of variance was used to partition variation in cannibalism rate into variability among half-sib families ('Sire'), full-sib families nested within sire ('Dam(Sire)'), and error. A significant 'Sire' effect indicates the presence of additive genetic variation in cannibalism rate, whereas a significant 'Dam' effect suggests the presence of nonadditive genetic variation or maternally caused variation (Falconer, 1989).

\section{Results}

\section{Experiment 1. Relative cannibalism of eggs and pupae}

Despite previous reports of Tribolium larvae eating pupae (e.g. Park et al., 1965), few pupae were consumed by the Chicago-cSM larvae tested here, although the rate of pupal cannibalism for larvae in oat flour was significantly greater than was the observed pupal cannibalism in wheat5 (mean per-capita consumption on oat: 0.15 pupa; on wheat5: 0.02 pupa; $P<0.0002$ ).

In contrast, eggs were readily consumed by the test larvae. Larval groups that had been reared in oat flour ate nearly all of the eggs that were provided (Fig. 2), whereas the larvae reared in wheat flour ate very few eggs (not significantly different from the control, $P>0.8)$. This experiment revealed that per-capita cannibalism by Chicago-cSM in the stressful oat environment was five times as great as in the optimal wheat5 environment $($ mean $=5.1$ eggs/individual in oat; mean $=0.98$ eggs/individual in wheat5; $P<0.0002$ ).

\section{Experiment 2. Egg cannibalism in four flours by Chicago-cSM}

When the egg cannibalism rate of groups of ChicagocSM larvae was tested further in four different flour

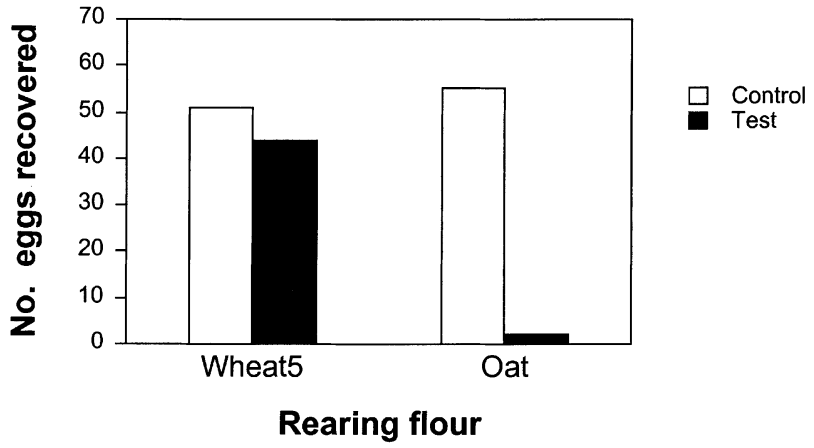

Fig. 2 Cannibalism by groups of Chicago-cSM Tribolium castaneum larvae in two flours. Each bar is the mean of five replicate trials in which groups were provided with 60 victim eggs for $48 \mathrm{~h}$ ('test'). In controls, no larvae were present in order to control for egg loss during sifting at the end of the trial.

types (wheat5, maize, rice and oat), there was again significantly greater egg eating when larvae were reared in oat (Table 1). Cannibalism rates in maize and rice were intermediate, as was performance in these flours (Via, 1991), consistent with the hypothesis that egg eating increases with the level of environmental stress.

\section{Experiment 3. Will the opportunity to cannibalize rescue larvae from the adverse effects of a poor environment?}

When sib groups of Chicago-cSM were allowed to eat eggs during development in oat flour, they were rescued from the adverse demographic effects of oat flour on development time, larval survival and early fecundity.

The mean development time was significantly more rapid for larvae in the oat + egg treatment $(\mathrm{O}+\mathrm{E})$ than for larvae reared in oat flour that was not supplemented with eggs $(\mathrm{O})$ (mean $=28.5$ days with eggs; mean $=44.8$ days without; $P<0.01$ ). The dis-

Table 1 Cannibalism rates for Chicago-cSM Tribolium castaneum tested on four flours. Entries are per-capita means for five vials each started with 20 eggs. These potential cannibals were allowed to grow until larval development was $\approx 75 \%$ complete and were then given a $48 \mathrm{~h}$ exposure to 60 victim eggs. Groups that share the same letter are not different at the $P<0.05$ level

\begin{tabular}{lcc}
\hline & \multicolumn{2}{c}{$\begin{array}{c}\text { Number of eggs } \\
\text { consumed per-capita }\end{array}$} \\
\hline Oat & 3.5 & $\mathrm{a}$ \\
Maize & 3.2 & $\mathrm{ab}$ \\
Rice & 2.7 & $\mathrm{bc}$ \\
Wheat5 & 2.3 & $\mathrm{c}$ \\
\hline
\end{tabular}

(C) The Genetical Society of Great Britain, Heredity, 82, 267-275. 
Table 2 Effect of adding weekly doses of eggs to oat flour on the average early fecundity and survivorship of Chicago-cSM Tribolium castaneum larvae. Groups that share the same letter are not different at the $P<0.05$ level

\begin{tabular}{llclc}
\hline & $\begin{array}{l}\text { Fecundity in } \\
\text { first } 6 \text { days }\end{array}$ & $\begin{array}{l}\text { Survival to } \\
\text { day } 13(\%)\end{array}$ \\
\hline $\begin{array}{l}\text { Treatment (flour type) } \\
\text { Wheat5 }\end{array}$ & 119 & $\mathrm{a}$ & 87.5 & $\mathrm{a}$ \\
Oat + eggs $(\mathrm{O}+\mathrm{E})$ & 112 & $\mathrm{ab}$ & 72.5 & $\mathrm{ab}$ \\
Oat $(\mathrm{O})$ & 105 & $\mathrm{~b}$ & 45 & $\mathrm{~b}$ \\
\hline
\end{tabular}

tribution of minimum development times (date that the first of the four individuals in each vial eclosed) among the 20 families in the $\mathrm{O}$ and $\mathrm{O}+\mathrm{E}$ treatments (Fig. 3) reveals the dramatic effects that egg eating had on development time in oat flour. Although both the mean and the minimum development time in the $\mathrm{O}+\mathrm{E}$ treatment were still significantly longer than in wheat5 (both at $P<0.05$ ), Fig. 3 shows that the modal minimum eclosion date was only one day later in $\mathrm{O}+\mathrm{E}$ than in the standard environment (wheat5). Development time decreased linearly with the number of eggs consumed per capita (Fig. 4), providing further evidence that egg eating provides an individual benefit to a cannibalistic Tribolium larva in oat flour.

In addition, both the survival and fecundity of individuals in the $\mathrm{O}+\mathrm{E}$ treatment were nominally higher than that of individuals of the same families in unsupplemented oat flour and they were not significantly lower than the values for individuals reared in wheat 5 (Table 2).

An analysis of variance of egg eating among the 20 families in the oat + eggs treatment revealed significant variance among full-sib families. This suggests that there is genetic variability in cannibalistic behaviour for larvae that are reared with the opportunity to cannibalize in a poor environment (Table 3).
Table 3 Genetic variation in egg eating within Chicago-cSM Tribolium castaneum larvae reared in oat flour with the opportunity to eat eggs during development

\begin{tabular}{lcccc}
\hline Source & d.f. & SS & $F$ & $P$ \\
\hline Family & 16 & 458 & 2.75 & 0.02 \\
Error & 17 & 177 & & \\
\hline
\end{tabular}

\section{Experiment 4. Egg eating by groups of Purdue +2 larvae raised on different flours}

When Purdue +2 larvae reared in different flours were allowed to eat eggs in a one-time trial, the ranking of cannibalism rates across flours was the same as that of the Chicago-cSM strain (compare Table 4 with Table 1). Approximately twice as many eggs were eaten per capita by Purdue +2 larvae that had been raised in oat flour than were consumed by larvae raised in wheat5. This experiment thus reveals that both strains are plastic in their egg-eating behaviour, with more eggs eaten in the more stressful environments.

\section{Experiment 5. Genetic variation in egg eating behaviour in Purdue +2}

The analyses of variance revealed highly significant variation among half-sib families in both wheat 5 and maize flour (the 'Sire' effect), and a significant 'Dam' effect in oat flour (Table 5). This means that for larvae reared in wheat 5 and maize, there is additive genetic variability for cannibalism behaviour. For larvae reared in oat with no eggs, the significant Dam effect suggests the presence of additive or nonadditive genetic variance (Falconer, 1989), given that maternal effects on larval egg-eating seem unlikely to be passed through the egg and persist to day 25 of larval life. Although the Sire effect was not significant in oat flour, the possibility that some additive genetic variance for egg eating was
Fig. 3 Distributions of the minimum development time of Chicago-cSM Tribolium castaneum larvae reared in groups of four in three different flour treatments (described in text). Wheat5 is the standard environment to which these strains are adapted. Oat flour is a novel environment, and in the oat + eggs treatment, 50 eggs from the stock culture were added each week as victims.

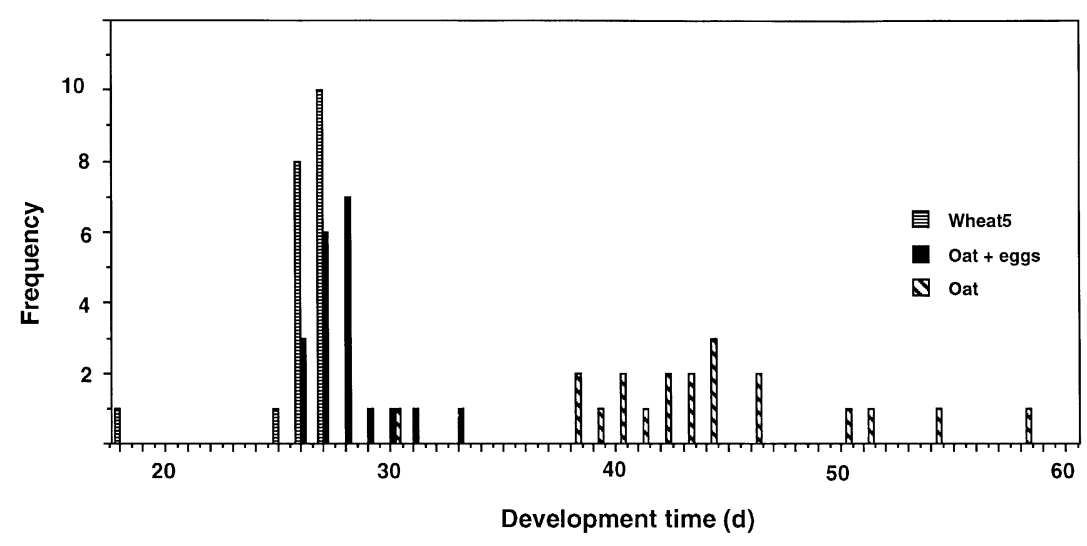

(C) The Genetical Society of Great Britain, Heredity, 82, 267-275. 


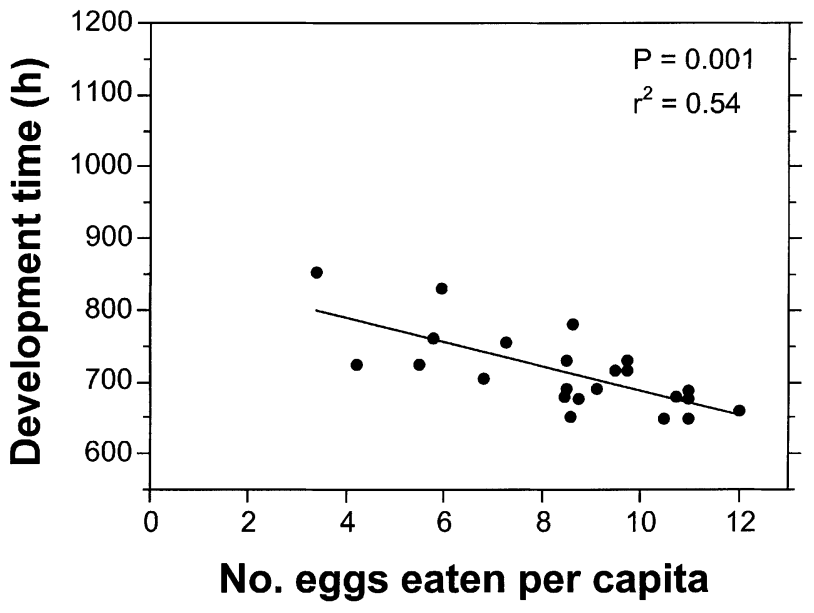

Fig. 4 Relationship between the number of eggs eaten per capita by Chicago-cSM Tribolium castaneum larvae in oat flour and the minimum development time in a vial of four individuals. Per capita egg consumption was calculated by dividing the number of eggs consumed by the number of surviving larvae in a given vial.

present among larvae reared in oat cannot be eliminated in this rather small experiment.

\section{Discussion}

For $T$. castaneum, the flour type in which an individual is immersed is a major defining feature of the environment, comparable to a host plant for a phytophagous insect. For laboratory strains of these beetles, including both of the strains of tested here, whole wheat flour with $5 \%$ brewer's yeast (wheat5) appears to be the best medium yet tested (Sokoloff et al., 1966a,b; Via, 1991). Before the start of this experiment, the two strains tested here had been reared for several hundred generations in wheat5 (Via, 1991), so other flours can be considered to be novel environments for these strains. Oat flour appears to be a particularly stressful new environment (Fig. 1; and Via, 1991; Via \& Conner, 1995).

When groups of larvae were offered a single opportunity to eat eggs when larval development was $\approx 75 \%$ complete, egg eating was consistently greater for larvae of both strains that had developed in oat flour than it was for individuals developing in wheat5 (Tables 1 and 4). Although absolute estimates of per-capita egg eating over a 2-day test period varied among experiments, the ranking of cannibalism rates in the different flours remained the same, with most eggs eaten in oat flour, fewest eaten in wheat5 and intermediate cannibalism rates in maize and rice flours. Thus, cannibalism of eggs by larvae is a phenotypically plastic behaviour that increases when individuals develop in a stressful environment.
When Chicago-cSM larvae reared in oat flour were provided with weekly doses of eggs to eat, they were rescued from the adverse effects of that stressful environment. Both survival and fecundity on oat flour were increased in the group of larvae that were allowed to eat eggs (Table 2). In addition, the development time of the first individual to eclose from each vial was dramatically reduced by the opportunity to cannibalize (Fig. 3). The inverse relationship between the number of eggs eaten and development time (Fig. 4) provides additional evidence that egg eating confers an individual nutritional advantage.

Significant genetic variation in cannibalism was seen in oat flour for Chicago-cSM larvae that had been offered eggs during the entire larval period (Table 3). However, genetic variation was only marginally significant (Purdue +2 ) for individuals reared in unsupplemented oat flour. This difference may have been caused either by a strain effect, or by an equally increased tendency to eat eggs among all families in the very stressful oat environment. The availability of eggs throughout the larval period in the oat + eggs treatment may have moderated the nutritional deprivation enough for genetic variation in egg-eating behaviour to be expressed.

Although several previous studies have demonstrated the presence of genetic variation in cannibalism in $T$. castaneum, none has used sib analyses for estimating the genetic variation in cannibalism, nor have other studies considered cannibalism in other than the standard environment. Park et al. (1965) showed that strains that maintain themselves at very different equilibrium densities cannibalize at different rates, with the smallest population being the most voracious. Stevens (1989) used inbred lines from several strains to document within-strain genetic variability for cannibalistic behaviour. Here, I show that cannibalism not only differs in intensity in different flours, but that there is significant genetic variation in cannibalism in several of the alternate environments.

Stevens (1989) interpreted the genetic variation in cannibalism that she found among strains growing in wheat5 as evidence for multiple stable optima in cannibalism. This interpretation hinges on her assertion that there is no selective benefit to cannibalism in wheat5. This is clearly not the case in oat flour. When there is an individual nutritional benefit to the cannibal, as seen here in oat flour, the cannibalism rate is likely to evolve to a single optimum value (Hamilton, 1964a,b). In such a case, the optimum presumably reflects a balance between the individual nutritional benefits of cannibalism and the reduced survival of progeny and availability of mates in highly cannibalistic strains. This optimum may vary among strains and depend on how 
Table 4 Cannibalism rates in Purdue +2 Tribolium castaneum tested on four flours. Conditions are as in Table 1. Groups that share the same letter are not different at the $P<0.05$ level

\begin{tabular}{lcc}
\hline & \multicolumn{2}{c}{$\begin{array}{c}\text { Number of eggs } \\
\text { consumed per-capita }\end{array}$} \\
\hline Oat & 2.9 & $\mathrm{a}$ \\
Maize & 2.1 & $\mathrm{ab}$ \\
Rice & 1.4 & $\mathrm{ab}$ \\
Wheat5 & 1.1 & $\mathrm{~b}$ \\
\hline
\end{tabular}

stressful a particular strain finds a new environment to be. Selection at the group level may also play a role in defining the optimum, because highly cannibalistic groups can drive themselves extinct (Park et al., 1964).

\section{Cannibalism and the colonization of new environments}

Despite the small size of the experiments, the genetic variation in cannibalism behaviour seen here suggests that the potential exists for evolution of an enhanced cannibalism rate upon entry into a novel and stressful environment such as oat flour. This result is highly relevant to the controversy about whether host shifts in phytophagous insects are accomplished primarily through evolutionary changes in behaviour (host finding, host acceptance) or by physiological adaptation (increases in survival and fecundity or decreases in development time of larvae) (e.g. Futuyma \& Moreno, 1988). An evolutionary increase in cannibalism upon entry into a new environment provides an additional way in which a behavioural change may facilitate colonization of a new and marginal habitat for populations that are relatively unadapted physiologically.

One aspect of this hypothesis that must be addressed is whether egg production in the stressful environment is sufficient to sustain a population of cannibalistic larvae during a range expansion. Although individual fecundity of Chicago-cSM females reared in oat flour is less than that for females reared in the standard environment (mean $=105$ eggs in the first 6 days of adult life in oat flour compared to 119 eggs in wheat5, Table 2), this number is greatly in excess of the number of larvae that will survive to adulthood. Therefore, eggs are likely to be available as a nutritional supplement to larvae growing in oat flour.

The observation that cannibalism can ease the adverse effects of a stressful environment does not imply the absence of natural selection for individuals with enhanced physiological adaptation to the new environment. None of the demographic components of fitness was improved by cannibalism to the level enjoyed by larvae reared in the standard environment, illustrating that there is still room for improvement in this new environment. In other experiments (Via \& Conner, 1995), genetic variation in demographic traits within oat flour was documented, suggesting that physiological adaptation upon prolonged exposure to oat flour is likely to occur.

The actual course of evolution within Chicago-cSM populations exposed to oat flour for a long period of time is likely to be determined by (a) the relative availability of genetic variance for cannibalism and physiological adaptation, and (b) the balance between the costs of an enhanced cannibalism rate, the nutritional benefits of increased cannibalism, and the fitness advantage of physiological adaptation to the new environment. If the population structure was such that selection also operated at the group level (e.g. Breden \& Wade, 1989; McCauley, 1992), predicting the outcome of exposure to oat flour would be even more difficult. Perhaps enhanced cannibalism might be a transient phenomenon in the new environment, with cannibalism rates increasing initially and then declining as physiological adaptation increases and the nutritional benefits of cannibalism thereby diminish.

Clearly, too great an increase in cannibalism in a novel environment could lead to extinction. Park et al. (1964) witnessed extinctions of some highly cannibalistic Tribolium populations, and Sokoloff et al. (1965) suggested that $T$. castaneum populations went extinct by excess

Table 5 Genetic variation in per capita egg cannibalism for Purdue +2 Tribolium castaneum on three flours. See text for experimental design. In this nested random-effects model, the Sire effect was tested over the nested Dam term. Mean values for each flour are found in Table 4

\begin{tabular}{|c|c|c|c|c|c|c|c|c|c|c|c|c|}
\hline Source & \multicolumn{4}{|c|}{ Wheat5 } & \multicolumn{4}{|c|}{ Maize } & \multicolumn{4}{|c|}{ Oat } \\
\hline Dam (Sire) & 61 & 434 & 0.7 & NS & 62 & 783 & 0.6 & NS & 61 & 209 & 1.6 & 0.01 \\
\hline Error & 139 & 1506 & & & 142 & 3227 & & & 132 & 278 & & \\
\hline$r^{2}$ & 0.38 & & & & 0.3 & & & & 0.5 & & & \\
\hline
\end{tabular}

(c) The Genetical Society of Great Britain, Heredity, 82, 267-275. 
cannibalism in maize flour after the competitor $T$. confusum (and its eggs, which served as alternative food) were eliminated by cannibalism-mediated competition. However, Dawson (1968) later asserted that, although egg eating appeared to benefit $T$. castaneum larvae in maize flour, it is more likely that the density-dependence of cannibalism will prevent population 'suicide' by declining when populations reach extremely low levels. As further evidence that cannibalism rates will not increase to lethal levels in the stressful oat flour environment, I maintained healthy laboratory populations of both the Chicago-cSM and the Purdue +2 strains in oat flour without any egg supplementation for over 3 years (Via, unpublished data). Unfortunately, the cannibalism rates in these cultures were not evaluated over time.

In conclusion, the data presented here provide some of the first available evidence that cannibalism behaviour may facilitate the colonization of a marginal new environment by rescuing individuals from a nutritionally poor situation. Further theoretical work is required in order to determine likely scenarios for the interplay between cannibalistic behaviour and physiological evolution in the process of adaptation to marginal environments. More empirical work would be useful in order to determine whether the possible facilitation of range expansion afforded by cannibalism might also accrue to some of the many other species that practise cannibalistic behaviour.

\section{Acknowledgements}

I am grateful to Susan Huckle for careful technical assistance. Funds for the experiments were provided by an award from the Searle Scholars Program (Chicago Community Trust), and a grant from NIH (R01GM34523). Final manuscript preparation was supported by NSF (DEB-9767573). I thank David Hawthorne for insightful comments on a previous version of the manuscript.

\section{References}

AGARWALA, B. K. AND DIXON, A. F. G. 1992. Laboratory study of cannibalism and interspecific predation in ladybirds. Ecol. Entomol., 17, 303-309.

BREDEN, F. AND WADE, M. J. 1989. Selection within and between kin groups of the imported willow leaf beetle. Am. Nat., 134, $35-50$.

DAwson, P. S. 1968. Xenocide, suicide and cannibalism in flour beetles. Am. Nat., 102, 97-105.

EICKWORT, K. R. 1973. Cannibalism and kin selection in Labidomera clivicollis (Coleoptera: Chrysomelidae). Am. Nat., 107, 452-453.

FALCONER, D. S. 1989. Introduction to Quantitative Genetics, 3rd edn. John Wiley and Sons, New York.
FINCKE, O. M. 1994. Population regulation of a tropical damselfly in the larval stage by food limitation, cannibalism, intraguild predation and habitat drying. Oecologia, 100, $118-127$.

FOX, L. R. 1975. Cannibalism in natural populations. Ann. Rev. Ecol. Syst., 6, 87-106.

FUTUYMA, D. J. AND MORENO, G. 1988. The evolution of ecological specialization. Ann. Rev. Ecol. Syst., 19, 207-233.

HAMILTON, W. D. 1964a. The genetical evolution of social behaviour. I. J. Theor. Biol., 7, 1-16.

HAMILTON, W. D. 1964b. The genetical evolution of social behaviour. II. J. Theor. Biol., 7, 17-52.

HASTINGS, A. and COSTANTINO, R. F. 1991. Oscillations in population numbers: age-dependent cannibalism. J. Anim. Ecol., 60, 471-482.

HO, F. K. AND DAWSON, P.S. 1966. Egg cannibalism by Tribolium larvae. Ecology, 47, 318-322.

JOHANSSON, G. 1992. Effects of zooplankton availability and foraging mode on cannibalism in three dragonfly larvae. Oecologia, 91, 179-183.

JOHANSSON, G. 1996. The influence of cannibalism and prey density on growth in the damselfly Coenagrion hastulatum. Arch. für Hydrobiol., 137, 523-535.

LLOYD, M. 1968. Self regulation of adult numbers by cannibalism in two laboratory strains of flour beetles (Tribolium castaneum). Ecology, 49, 245-259.

McCAULEY, D. E. 1992. Family structured patterns of mortality in the false Colorado potato beetle. Ecol. Entomol., 17, 142148.

MERTZ, D. B. AND ROBERTSON, J. R. 1970. Some developmental consequences of handling, egg-eating and population density for flour beetle larvae. Ecology, 51, 990-998.

NASEER, M. AND ABDURAhimAN, U. C. 1993. Cannibalism in Cardiastethus exiguus Poppius (Hemiptera: Anthocoridae): A predator of the coconut caterpillar Opsina arenolella Walker (Lepidoptera: Xylorictidae). J. Adv. Zool., 14, 1-6.

PARAJUleE, M. N. AND PHILlips, T. W. 1995. Survivorship and cannibalism in Lyctocoris campestris (Hemiptera: Anthocoridae): Effects of density, prey availability and temperature. J. Entomol. Sci., 30, 1-8.

PARK, T. 1948. Experimental studies of interspecies competition. I. Competition between populations of the flour beetles Tribolium confusum Duv. and Tribolium castaneum Herbst. Ecol. Monogr., 18, 265-308.

PARK, T. 1962. Beetles, competition and populations. Science, 138, 1369-1375.

PARK, T., LESLIE, P. H. AND MERTZ, D. B. 1964. Genetic strains and competition in populations of Tribolium. Physiol. Zool., 37, 97-162.

PARK, T., MERTZ, D. B., GRODZINSKI, W. AND PRUS, T. 1965. Cannibalistic predation in populations of flour beetles. Physiol. Zool., 38, 289-321.

PARK, T., MERTZ, D. B. AND NATHANSON, M. 1968. The cannibalism of pupae by adult flour beetles. Physiol. Zool., 41, 228-253.

POLIS, G. A. 1981. The evolution and dynamics of intraspecific predation. Ann. Rev. Ecol. Syst., 12, 225-251.

RICH, E. R. 1956. Egg cannibalism and fecundity in Tribolium. Ecology, 37, 109-120. 
SOKOLOFF, A., LERNER, M. AND HO, F. K. 1965. Self-elimination of Tribolium castaneum following xenocide of T. confusum. Am. Nat., 99, 399-404.

SOKOLOFF, A., FRANKLIN, I. R. AND LAKHANPAL, R. K. 1966 a. Comparative studies with Tribolium (Coleoptera: Tenebrionidae). II. Productivity of $T$. castaneum Herbst and $T$. confusum Duval on natural semi-synthetic and synthetic diets. J. Stored Prod. Res., 1, 313-324.

SOKOLOFF, A., FRANKLIN, I. R., OVERTON, L. F. AND HO, F. K. 1966b. Comparative studies with Tribolium (Coleoptera: Tenebrionidae). I. Productivity of $T$. castaneum Herbst and $T$. confusum Duval on several commercially available diets. J. Stored Prod. Res., 1, 295-311.

SONLEITNER, F. J. 1961. Factors affecting egg cannibalism and fecundity in populations of adult Tribolium castaneum Herbst. Physiol. Zool., 34, 233-255.

SONLEITNER, F. J. AND GUTHRIE, P. J. 1991. Factors affecting oviposition rate in the flour beetle Tribolium castaneum and the origin of the population regulating mechanism. Res. Pop. Ecol., 33, 1-12.

SPENCE, J. R. AND CARCAMO, H. A. 1991. Effects of cannibalism and intraguild predation on pondskaters (Gerridae). Oikos, 62, 333-341.

STEVENS, L. 1989 . The genetics and evolution of cannibalism in flour beetles (genus Tribolium). Evolution, 43, 169-179.
TSCHINKEL, W. R. 1993. Resource allocation, brood production and cannibalism during colony founding in the fire ant, Solenopsis invicta. Behav. Ecol. Sociobiol., 33, 209- 233.

VAN BUSKIRK, J. 1989. Density-dependent cannibalism in larval dragonflies. Ecology, 70, 1442-1449.

VIA, S. 1991. Variation between strains of the flour beetle Tribolium castaneum in relative performance on five flours. Entomologia exp. appl., 60, 173-182.

VIA, S. AND CONNER, J. 1995. Evolution in heterogeneous environments: genetic variability within and across different grains in Tribolium castaneum. Heredity, 74, 80-90.

WADE, M. J. 1980. An experimental study of kin selection. Evolution, 34, 844-854.

WAGNER, J. D. AND wISE, D. H. 1996. Cannibalism regulates densities of young wolf spiders: evidence from field and laboratory experiments. Ecology, 77, 639-652.

WATANABE, M. AND YAMAGUCHI, H. 1993. Egg cannibalism and egg distribution of two Pieris butterflies, Pieris rapae and P. melete (Lepidoptera: Pieridae) on a host plant, Rorippa indica (Cruciferae). Jap. J. Ecol., 43, 181-188.

WISSINGER, S. A., SPARKS, G. B., ROUSE, G. L., BROWN, W. S. AND STELTZER, H. 1996. Intraguild predation and cannibalism among larvae of detritivorous caddisflies in subalpine wetlands. Ecology, 77, 2421-2430. 